\title{
Psychological reluctance to insulin therapy: as an illness side of diabetes mellitus
}

\author{
Narihito Yoshioka ${ }^{1}[$
}

Received: 26 December 2017 / Published online: 17 February 2018

(c) The Japan Diabetes Society 2018

In the earlier stages of type 2 diabetes mellitus, the disease can be managed with lifestyle modification with or without oral anti-diabetic drugs (OADs). However, because of the progressive nature of the disease caused by the gradual reduction of functional $\beta$-cell mass, many patients will require insulin therapy to maintain adequate glycemic control. Despite the clinical benefits of timely initiation of insulin therapy, it is often delayed as a result of clinical inertia and resistance by patients. The term "clinical inertia" is defined as failure of health care providers to initiate or intensify therapy when indicated. In a survey of patients with type 2 diabetes who attended general practitioners in the United Kingdom, $25 \%$ of patients had insulin initiation delayed for at least 1.8 years and $50 \%$ of patients had delayed for 5 years after failure of glycemic control with OADs [1].

Patient reluctance to receive insulin therapy is due primarily to psychological insulin resistance (PIR), which can be defined as psychological opposition to insulin use [2,3]. Many patients have concerns, including misconceptions, about insulin therapy. PIR includes the belief that insulin is ineffective, the belief that starting insulin is a sign of failure, fear of injection pain, and fear of hypoglycemia. PIR also exists in the side of insulin prescribers. Ishii et al. had demonstrated that the mean $\mathrm{HbA1c}$ at which physicians responded by actually recommending insulin to their patients was $9.6 \%$, while they would reduce this level to $8.2 \%$ if they themselves required insulin [4]. Physicians could not initiate insulin treatment at the timing they would start. Physician barriers to initiate insulin were lack of staff (nurses, pharmacists) who could assist with explanation, concern about the

Narihito Yoshioka

n.yoshioka@east.ntt.co.jp

1 Division of Diabetes and Endocrinology, Department of Medicine, Sapporo Medical Center, NTT East Corporation, Minami 1-jyo, Nishi 15-chome, Chuo-ku, Sapporo 060-0061, Japan use of insulin in elderly patients and difficulties to provide guidance and education on insulin injection to patients [4].

In our previous study, patients most frequently reported that they did not want to inject themselves with insulin forever [5]. Historically, insulin has been an underutilized "last resort" option in diabetic management. However, it is becoming increasingly valued safe with aggressive progresses both of insulin derivatives itself and devices to inject insulin. Insulin injections do not always continue for the rest of a patient's lifetime. The gap exists in perceptions concerning initiation of insulin therapy between patients and physicians. Physicians underestimated patient attitudes related to social and interpersonal effects, such as, "I don't want to be different from others", "I don't want others to know", "My friendship may suffer" and "My family will be worried". The only statement that physicians significantly overestimated in importance was, "Injections are painful". The medical profession has traditionally focused on clinical or physical aspects rather than psychosocial outcomes, and this may be one possible explanation for under- or over-estimation of psychosocial barriers by physicians. What physicians believe to be the concerns of patients regarding insulin initiation, and what actually concerns patients in relation to insulin, may not be the same. To facilitate insulin uptake, health care providers, therefore, need to be educated regarding potential social and interpersonal fears surrounding insulin use.

As a disease, diabetes mellitus is a group of metabolic disease characterized by hyperglycemia resulting from defects in insulin secretion, insulin action or both. However, as an illness, diabetes suffers many burdens to patients and their surroundings. We as a medical profession, have to understand diabetes from both aspects, as a disease and as an illness.

Being a specialist in diabetes does not necessarily guarantee an appropriate recognition of patients' concerns. This may be due primarily to the lack of time allotted for examinations, making it difficult for physicians to understand and address individual causes of anxieties about diabetes 
management. Nevertheless, it is increasingly recognized that psychosocial factors are inherent to diabetes and its successful treatment. Clearly, it is useful to develop strategies to identify and reduce psychological insulin resistance. The first step to overcoming patient anxiety about insulin therapy is to identify the specific cause of anxiety for a particular individual.

William Osler (1849-1919) stated, "It is much more important to know what sort of a patient has a disease, than what sort of a disease a patient has."

\section{Compliance with ethical standards}

Conflict of interest The author declares there is no conflict of interest associated with the manuscript.

\section{References}

1. Rubino A, McQuay LJ, Gought SC, et al. Delayed initiation of subcutaneous insulin therapy after failure of oral glucose-lowering agents in patients with type 2 diabetes: a population-based analysis in the UK. Diabet Med. 2007;24:1412-8.

2. Polonsky WH, Fisher L, Guzma S, et al. Psychological insulin resistance in patients with type 2 diabetes. Diabetes Care. 2005;28:2543-5.

3. Brod M, Kongso JH, Lessard S, et al. Psychological insulin resistance: patient beliefs and implications for diabetes management. Qual Life Res. 2009;18:23-32.

4. Ishii H, Iwamoto Y, Tajima N. An explanation of barriers to insulin initiation for physicians in Japan: findings from the diabetes attitude, wishes and needs (DAWN) Japan study. PLoS ONE. 2012;7:e36361.

5. Yoshioka N, Ishii H, Tajima N, et al. Differences in physician and patient perceptions about insulin therapy for management of type 2 diabetes: the DAWN Japan study. Curr Med Res Opin. 2014;30:177-83. 